\title{
Effectiveness of Chitosan Films Impregnated With Ciprofloxacin for the Prophylaxis of Osteomyelitis in Open Fractures: An Experimental
} Study in Rats

\author{
Lincoln Paiva Costa, ${ }^{1,}$ Luiz Eduardo Moreira Teixeira, ${ }^{2}$ Gustavo Silame Maranhão Lima, ${ }^{3}$ Marcelo \\ Mendes Ferreira, ${ }^{1}$ Mateus Antunes de Andrade, ${ }^{1}$ Paula Vieira Teixeira Vidigal, ${ }^{4}$ André Augusto Gomes \\ Faraco, ${ }^{4}$ Eduardo Frois Temponi, ${ }^{1}$ and Ivana Duval de Araújo ${ }^{4}$ \\ ${ }^{1}$ Hospital Madre Teresa, Belo Horizonte, Brazil \\ ${ }^{2}$ Hospital Madre Teresa and Universidade Federal de Minas Gerais Minas Gerais, Belo Horizonte, Brazil \\ ${ }^{3}$ Hospital das Clínicas da Universidade Federal de Minas Gerais, Belo Horizonte, Brazil \\ ${ }^{4}$ Universidade Federal de Minas Gerais Minas Gerais, Belo Horizonte, Brazil \\ "Corresponding author: Lincoln Paiva Costa, Hospital Madre Teresa, Belo Horizonte, Brazil. Tel: +55-31987750451, E-mail: lincolnpaivacosta@yahoo.com.br
}

Received 2016 February 06; Revised 2016 May 05; Accepted 2016 May 10.

\begin{abstract}
Background: The systemic antimicrobial prophylaxis is the standard treatment in the prevention of osteomyelitis after open fractures, with topical application of antimicrobials as an alternative due to their high concentrations at the site of the fracture, low systemic concentrations and fewer side effects.

Objectives: This study aimed to evaluate the effectiveness of prophylaxis of osteomyelitis through experimental model of open fractures with the use of chitosan films, whether or not impregnated with ciprofloxacin.

Materials and Methods: In this experimental study, 24 Holtzman rats were distributed into 4 groups of 6 rats each. The CT (control of treatment) group: an open fracture model treated with systemic antimicrobial; the IC (infection control) group: an open fracture untreated model; the C (chitosan) group: an open fracture model treated using a chitosan film; and the CA (chitosan with antimicrobial) group: an open fracture model treated using a chitosan film impregnated with antimicrobial. After 3 weeks the animals were killed by an overdose of anesthetic, and a fragment osseous was removed for histological and microbiological analysis. The comparisons between the groups considered significant values of $\mathrm{P} \leq 0.05$.

Results: In cultures of the CT group, there was less bacterial growth compared to the results of the cultures of the IC $(\mathrm{P}=0.005), \mathrm{C}$ $(\mathrm{P}=0.005)$ and $\mathrm{CA}(\mathrm{P}=0.009)$ groups. The inflammation was lower in the CT group compared to the $\mathrm{IC}(\mathrm{P}=0.014), \mathrm{C}(\mathrm{P}=0.001)$ and $\mathrm{CA}(\mathrm{P}=0.007)$ groups.

Conclusions: In this experimental model of open fracture, the chitosan film pure or impregnated with ciprofloxacin was not effective in the prophylaxis of osteomyelitis.

Keywords: Open Fracture, Prophylaxis, Osteomyelitis, Chitosan, Ciprofloxacin
\end{abstract}

\section{Background}

The incidence of open fractures in long bones is estimated 11.5 in 100.000 people (1); the purpose of the treatment is to prevent infection, promote fracture healing and restore function (2). Despite treatment, about 10\% to 50\% will progress to osteomyelitis (3-5). Systemic prophylaxis is the standard for the treatment of open fractures since 1974, and its benefit was confirmed by a Cochrane review, where it was observed that the use of antibiotics after open fractures reduces the risk of infection by $59 \%$ (6).

Over the last decade, it has increased trend for the application of topical antimicrobial prophylaxis of osteomyelitis secondary to open fractures (7). This method provides high concentration of an antimicrobial at the site of the fracture with low systemic concentration, reducing the risk of side effects (7). The most frequently used for this purpose, is the polymethylmethacrylate (PMMA). An alternative is chitosan, a biological product derived from the chitin of shellfish, bioabsorbable (8), bactericidal (9) and allowing storage and gradual release of drugs (10), including antimicrobials. Dehydrated chitosan films have the ability to rapidly rehydrate and absorb drugs (11). Manipulation of the properties of this film during production 
may improve the local distribution of assisting in the prophylaxis of antimicrobial infections $(10,11)$.

\section{Objectives}

The aim of this study was to evaluate the effectiveness of prophylaxis of osteomyelitis secondary to open fractures in an experimental model with chitosan films, whether or not impregnated with ciprofloxacin comparing with standard treatment with antibiotic prophylaxis.

\section{Materials and Methods}

Between January and August 2015, 24 males Holtzman rats selected from Universidade federal de minas Gerais (UFMG), with approximately three months of life and weighing $261.95 \pm 20.82$ were divided into 4 groups:

The control treatment $(\mathrm{CT})$ group $(\mathrm{n}=6)$ : open fracture treated with systemic antimicrobial.

The infection control (IC) group ( $n=6)$ : open fracture without treatment.

The chitosan (C) group $(n=6)$ : open fracture treated with a pure chitosan film.

The chitosan with antimicrobial (CA) group $(n=6)$ : open fracture treated with a chitosan film impregnated with ciprofloxacin.

The rats were placed in cages with three animals of the same group at the vivarium of the UFMG Medical School with water and rat food, temperature control, ventilation and natural day-night cycle under daily monitoring by the researchers. The animals were anesthetized and maintained under sedation by intraperitoneal injection of ketamine and xylazine at doses of $15 \mathrm{mg} / \mathrm{kg}$ and $60 \mathrm{mg} / \mathrm{kg}$, respectively. The femoral fracture of the left hind leg of the animal was performed using special equipment that makes the fracture with a similar pattern in all the animals (Figure 1). After the trichotomy on the left hand, antisepsis was done with polivinilpirrolidona (Povidine $₫$ - Johnson \& Johnson, Brazil) in degerming solution, followed by an alcoholic solution. The protection of the area with sterile drapes was done, keeping exposed the paw to be operated. After sterile surgical scrub was done, an incision was made longitudinal in the middle third of the left thigh and dissection by planes to exposure the femoral fracture. The intramedullary fixation of the fracture was performed using a needle aspiration $40 \times 1.2 \mathrm{~mm}$ and 0.1 milliliters of inoculated suspension of Staphylococcus aureus (UFMG Central $\mathrm{Lab}$ ) in concentration of $10 \mathrm{CFU} / \mathrm{mL}$ set by McFarland (12), applied in the bed of the fracture. The wound was sutured with nonabsorbable monofilament nylon 5-0 (Mononylon Ethicon $®)$.

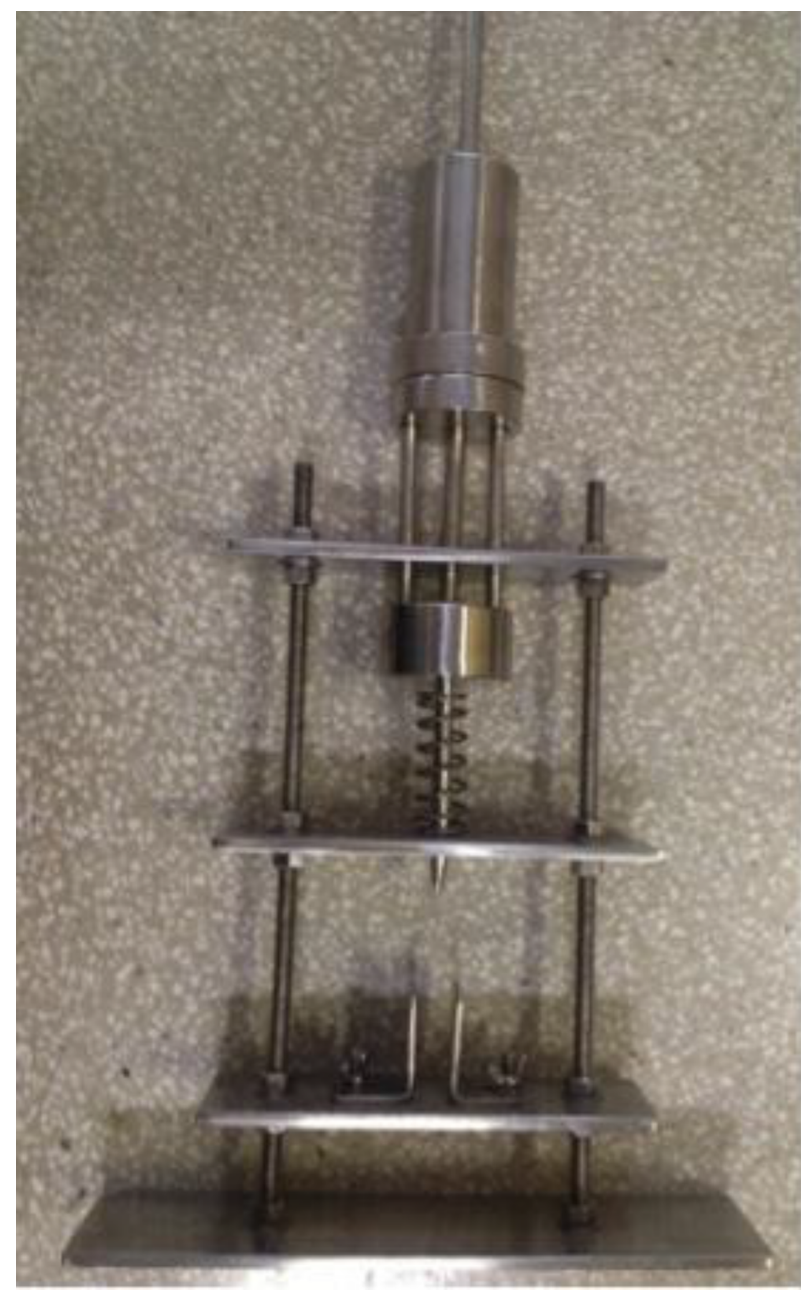

Figure 1. Special Equipment That Was Used to Make the Fracture

In the $\mathrm{C}$, and $\mathrm{CA}$ groups, the sterile chitosan film was placed in the bed of the fracture with dimensions of 0.5 $\mathrm{cm} \times 0.5 \mathrm{~cm}$ pure and impregnated with ciprofloxacin $10 \%$ (Shaanxi New LeaderTraiding Co. Ltd. China.) respectively, followed by the closure of the wound in previous groups. After surgery the animals were kept in the vivarium, with water and food. Analgesia was performed through solution of meloxicam (Mobic $($ - Boehringer Ingelheim Brazil Ltda, SP, Brazil) with a dose of $0.2 \mathrm{mg} / \mathrm{kg}$ subcutaneously on the immediate postoperative period.

The CT group was treated with ciprofloxacin (Ciprodez (B - Bio -Vet S/A, SP, Brazil) intraperitoneal dose of 2.5 $\mathrm{mg} / \mathrm{kg} /$ day, for three days and the IC group did not receive any treatment to open fracture. The wound was daily evaluated and the sutures were removed 10 days after the surgery. After three weeks, the animals were killed through 
anesthetic overdose under aseptic conditions, the femur was removed and the material submitted to qualitative culture and histological analysis.

The cultures were performed on blood agar and considered positive when showed bacterial growth in 72 hours with the same microorganism inoculated in the medullary bed (Staphylococcus aureus). In all groups qualitative cultures were performed in the suspension with $S$. aureus to ensure the presence of bacteria in the fracture of the femur of the studied animals. All S. aureus isolated were submitted to antibiogram to verify sensitivity to ciprofloxacin.

For histological examination, the samples were stained with hematoxylin-eosin and evaluated by the same pathologist in conventional optical microscopy researching osteomyelitis in activity. Histological classification was performed based on data for intraosseous acute inflammation, intraosseous chronic inflammation, periosteal inflammation and bone necrosis using the system of Smeltzer et al. (13). Each parameter was graded on a five-point scale (zero to four) and the sum of respective histological parameters calculated as a total histological score.

The study was approved by the ethics committee of animal use at Universidade federal de Minas Gerais (UFMG), under protocol 240/2011.

\subsection{Data Analysis}

The sample size was calculated using Minitab® version 14.1 software (Minitab Inc., PA, USA), and alpha $=0.05$, test power $>0.80$ and maximum deviation between the results equal to the average, resulting in six sample animals per group. The values of mean and standard deviation were established after performing a pilot project.

The data were analyzed by the software EPI inform 7.1.2.0 for Windows $®$ (Atlanta, USA) using Fisher's exact test to compare the qualitative variables and the MannWhitney test to compare quantitative variables. Weight difference between groups was detected by the Kruskal-Wallis test. Differences were considered significant with $\mathrm{P}<0.05$.

\section{Results}

There was no significant difference in the average weight of the animals between the groups (Table 1). A significant difference was observed between the cultures of the CT group compared with the cultures of the IC, C and CA groups (Table 2). The antibiogram showed sensitivity of the isolated bacteria to ciprofloxacin. Histological analysis showed a significant difference in the total points of animals undergoing treatment with systemic antibiotic prophylaxis (the CT group) compared to the other groups. It was also seen that when parameters of this scoring system were evaluated in isolation, the biggest difference between the animals of the group treated with antibiotic prophylaxis (the CT group) and the others was the degree of intraosseous chronic inflammation. The comparison of histological results is summarized in Table 3.

Table 1. Animals Weight in the Different Groups
\begin{tabular}{cccc}
\hline \multirow{3}{*}{ Group } & \multicolumn{3}{c}{ Animals Weight } \\
\cline { 2 - 4 } & Mean $(\mathbf{g})$ & SD $(\mathbf{g})$ & P Value \\
\hline CT & 252,50 & 20,29 & - \\
IC & 265,83 & 16,55 & 0,44 \\
C & 259,50 & 20,40 & 0,84 \\
CA & 270,00 & 26,13 & 0,56 \\
\hline
\end{tabular}

Abbreviations: SD, standard deviation; g, gram.

Table 2. Results of Qualitative Cultures in the Different Groups

\begin{tabular}{lccc}
\hline \multirow{2}{*}{ Group } & \multicolumn{2}{c}{ Culture Results } & \multirow{2}{*}{ PValue } \\
\cline { 2 - 3 } & Positive & Negative & \\
\hline CT & 0 & 6 & - \\
IC & 5 & 1 & 0.005 \\
C & 6 & 0 & 0.009 \\
CA & 5 & 1 & 0.005 \\
\hline
\end{tabular}

\section{Discussion}

The most important finding of this study was that a chitosan film impregnated with ciprofloxacin can be used for local delivery of antibiotics in open fractures wound; however, the concentrations of $10 \%$ of ciprofloxacin is not effective to prevent the development of infection in a rat model of open fracture.

The principles in the treatment of open fractures are the surgical emergency treatment, appropriate antibiotic therapy, adequate irrigation and debridement, stabilization of the fracture, wound healing and rehabilitation. Infection is the most common complication of open fractures and antibiotic therapy should be performed in all cases of open fractures $(4,5)$. The use of systemic antimicrobials has been considered the standard in the treatment of open fractures since 1974 when Patzakis et al. (14) observed that patients treated with cephalothin showed lower rates of osteomyelitis compared to patients treated with penicillin and streptomycin. The ideal time for treatment with antimicrobials is uncertain and most authors 
Table 3. Degree of Inflammation of Bone Fragment Through the Score Smeltzer

\begin{tabular}{|c|c|c|}
\hline Value & $\begin{array}{c}\text { Bone Histology } \\
\text { Mean Punctuation }\end{array}$ & PValue \\
\hline \multicolumn{3}{|l|}{ CT Group } \\
\hline Intraosseous acute inflammation & $0 \pm 0$ & - \\
\hline Intraosseous chronic inflammation & $1.0 \pm 0.6$ & - \\
\hline Periosteal inflammation & $1.5 \pm 1.6$ & - \\
\hline Bone necrosis & $1.7 \pm 0.4$ & - \\
\hline Total & $2.7 \pm 1.9$ & - \\
\hline \multicolumn{3}{|l|}{ IC Group } \\
\hline Intraosseous acute inflammation & $0.3 \pm 0.8$ & 0,34 \\
\hline Intraosseous chronic inflammation & $3.7 \pm 0.5$ & 0.001 \\
\hline Periosteal inflammation & $0 \pm 0$ & 0.049 \\
\hline Bone necrosis & $1.8 \pm 1.2$ & 0,74 \\
\hline Total & $5.8 \pm 1.7$ & 0.014 \\
\hline \multicolumn{3}{|l|}{ C Group } \\
\hline Intraosseous acute inflammation & $2.0 \pm 1.9$ & 0.027 \\
\hline Intraosseous chronic inflammation & $3.7 \pm 0.5$ & 0.001 \\
\hline Periosteal inflammation & $2.0 \pm 1.1$ & 0.54 \\
\hline Bone necrosis & $2.8 \pm 1.5$ & 0,09 \\
\hline Total & $10.5 \pm 3.1$ & 0.001 \\
\hline \multicolumn{3}{|l|}{ CA Group } \\
\hline Intraosseous acute inflammation & $1.5 \pm 1.2$ & 0,13 \\
\hline Intraosseous chronic inflammation & $3.5 \pm 0.5$ & 0.000025 \\
\hline Periosteal inflammation & $0.8 \pm 0.4$ & 0,35 \\
\hline Bone necrosis & $0.8 \pm 0.4$ & 0.005 \\
\hline Total & $6.7 \pm 2.2$ & 0.007 \\
\hline
\end{tabular}

recommend its use for up to three days after injury (15). Despite the intravenous use is still considered the standard, the local application of antibiotics seems as an alternative for prophylaxis of bone infections leading to high local concentrations of antibiotics and low systemic levels (16). The choice of the antimicrobial agent to be used is also controversial, with ciprofloxacin been an option in the treatment of open fractures due to its action against Grampositive and Gram-negative organisms (17).

In the present study, an experimental model of an open fracture was performed to evaluate the effectiveness of prophylaxis of infection in each group tested, verifying the occurrence of osteomyelitis. This model was suggested by Lindsey et al. (12), who proved an index of reproducible osteomyelitis of $90 \%$ to $100 \%$ after 21 days after the experimental fracture, the same was observed in this research, because five of the six animals in the IC group developed in- fection after a period of 21 days after surgery. Ciprofloxacin was used for acting appropriately in the prophylaxis of infections secondary to open fractures and was stable when combined with a chitosan film. The antibiotic was used for three days after the injury.

PMMA is the current standard vehicle for local antibiotics delivery in orthopedic surgery (18-21). However, it needs thermostable antibiotics, provides uncontrolled release of drugs and surgical removal is necessary because it is not biodegradable (21). A number of experimental studies have suggested the use of other options for local antibiotic delivery such as a chitosan film impregnated with antimicrobial (16, 21-25).

In this study, chitosan was used as a vehicle, because it has the ability to transport drugs like PMMA, with the additional benefit of possessing antibacterial activity (26) and be biodegradable $(8,16,21,25)$; so, a second surgical pro- 
cedure is not required to remove it from the wound. Besides, chitosan does not increase the temperature during the manufacturing process and can be combined with any antibiotic, not requiring drugs that have thermal stability.

Although Aimin et al. (27) describe the reduction of the infection rate of $S$. aureus osteomyelitis in an experimental model using pure chitosan; we did not get the same result. The animals of the $C$ group received pure chitosan films as a treatment for the model of open fracture and presented a high rate of osteomyelitis compared to the CT group that received antibiotic prophylaxis, with a significant difference. Histological evaluation also showed a higher degree of intraosseous chronic inflammation in the $\mathrm{C}$ group compared to the CT group, with a significant difference. This result may have been due to the small size of a chitosan film used in our research or extensive soft tissue injury caused by the equipment for inducing fracture that results in more favorable environment to infection.

Orhan et al. (22) evaluated the efficacy of chitosan microspheres and pectin impregnated with ciprofloxacin in a model of local treatment of osteomyelitis and concluded that this type of treatment was higher than the equivalent treatment intramuscular antimicrobial. Stinner et al. (16) evaluated the efficacy of a chitosan sponge impregnated with amikacin or vancomycin in a model of complex musculoskeletal wound and concluded that this treatment was effective in reducing the bacteria concentration within the wound. In this study, different results were obtained. The CA group exposed to the treatment of an open fracture model through a chitosan film impregnated with ciprofloxacin showed a high rate of osteomyelitis compared to the CT group submitted to antibiotic prophylaxis with a significant difference. This result can be justified by the small amount of antibiotic associated to a chitosan film used in this study where the preparation had $10 \%$ of the molecular weight of the membrane in antibiotic.

Further studies will be necessary to assess whether the membrane of chitosan impregnated with ciprofloxacin in higher concentrations of antibiotics or larger than that used in this study will be effective in the prophylaxis of osteomyelitis secondary to fractures. There are not studies in the literature using chitosan films impregnated with ciprofloxacin for the prophylaxis of osteomyelitis secondary to open fractures.

As a strong point this study bring a new method of the treatment for open fractures with local delivery of antibiotics through chitosan films resulting in higher local concentrations of drugs and lower systemic concentrations and lower side effects. The weak points of this study is that it is an experimental study, not tested in humans and used just the concentration of $10 \%$ of antibiotics in chitosan films that was not effective to avoid infection in this model of open fracture.

\subsection{Conclusion}

The chitosan film pure or impregnated with ciprofloxacin showed no efficacy in the prophylaxis of osteomyelitis.

\section{Acknowledgments}

The authors would like to thank the Universidade Federal de Minas Gerais for their support in conducting this study.

\section{Footnote}

Authors' Contribution: Lincoln Paiva Costa, Luiz Eduardo Moreira Teixeira and Ivana Duval de Araújo were responsible for collecting the data, and preparing the manuscript and participated in all phases of the study; Eduardo Frois Temponi and Gustavo Silame Maranhão Lima designed the research plan, supervised the analysis of the data and assisted in writing the manuscript; Marcelo Mendes Ferreira and Mateus Antunes de Andrade assisted in collecting the data and reviewing the literature; and Paula Vieira Teixeira Vidigal and André Augusto Gomes Faraco assisted in analyzing the data and writing the manuscript.

\section{References}

1. Court-Brown CM, Rimmer S, Prakash U, McQueen MM. The epidemiology of open long bone fractures. Injury. 1998;29(7):529-34. [PubMed: 10193496].

2. Okike K, Bhattacharyya T. Trends in the management of open fractures. A critical analysis.J Bone Joint Surg Am. 2006;88(12):2739-48. doi: 10.2106/JBJS.F.00146. [PubMed: 17142427].

3. Zalavras CG, Patzakis MJ. Open fractures: evaluation and management. J Am Acad Orthop Surg. 2003;11(3):212-9. [PubMed: 12828451].

4. Gustilo RB, Anderson JT. Prevention of infection in the treatment of one thousand and twenty-five open fractures of long bones: retrospective and prospective analyses.JBone Joint Surg Am. 1976;58(4):4538. [PubMed: 773941].

5. DeLong WJ, Born CT, Wei SY, Petrik ME, Ponzio R, Schwab CW. Aggressive treatment of 119 open fracture wounds. J Trauma. 1999;46(6):1049-54. [PubMed: 10372623].

6. Gosselin RA, Roberts I, Gillespie WJ. Antibiotics for preventing infection in open limb fractures. Cochrane Database Syst Rev. 2004(1):CD003764. doi: 10.1002/14651858.CD003764.pub2. [PubMed: 14974035].

7. Eckman JB, Henry SL, Mangino PD, Seligson D. Wound and serum levels of tobramycin with the prophylactic use of tobramycinimpregnated polymethylmethacrylate beads in compound fractures. Clin Orthop Relat Res. 1988;237:213-5.

8. Mi FL, Tan YC, Liang HF, Sung HW. In vivo biocompatibility and degradability of a novel injectable-chitosan-based implant. Biomaterials. 2002;23(1):181-91. [PubMed: 11762837]. 
9. Fujimoto T, Tsuchiya Y, Terao M, Nakamura K, Yamamoto M. Antibacterial effects of chitosan solution against Legionella pneumophila, Escherichia coli, and Staphylococcus aureus. Int J Food Microbiol. 2006;112(2):96-101. doi: 10.1016/j.ijfoodmicro.2006.06.003. [PubMed: 17045689].

10. Khor E, Lim LY. Implantable applications of chitin and chitosan. Biomaterials. 2003;24(13):2339-49. [PubMed:12699672].

11. Nunthanid J, Puttipipatkhachorn S, Yamamoto K, Peck GE. Physical properties and molecular behavior of chitosan films. Drug Dev Ind Pharm. 2001;27(2):143-57. doi: 10.1081/DDC-100000481. [PubMed: 11266226].

12. Lindsey BA, Clovis NB, Smith ES, Salihu S, Hubbard DF. An animal model for open femur fracture and osteomyelitis: Part I. J Orthop Res. 2010;28(1):38-42. doi: 10.1002/jor.20960. [PubMed: 19637274].

13. Smeltzer MS, Thomas JR, Hickmon SG, Skinner RA, Nelson CL, Griffith $\mathrm{D}$, et al. Characterization of a rabbit model of staphylococcal osteomyelitis.J Orthop Res. 1997;15(3):414-21. doi:10.1002/jor.1100150314. [PubMed: 9246088].

14. Patzakis MJ, Harvey JJ, Ivler D. The role of antibiotics in the management of open fractures. J Bone Joint Surg Am. 1974;56(3):532-41. [PubMed: 4150798].

15. Zalavras CG, Patzakis MJ, Holtom PD, Sherman R. Management of open fractures. Infect Dis Clin North Am. 2005;19(4):915-29. doi: 10.1016/j.idc.2005.08.001. [PubMed: 16297739].

16. Stinner DJ, Noel SP, Haggard WO, Watson JT, Wenke JC. Local antibiotic delivery using tailorable chitosan sponges: the future of infection control?. J Orthop Trauma. 2010;24(9):592-7. doi: 10.1097/BOT.ob013e3181ed296c. [PubMed: 20736801].

17. Patzakis MJ, Bains RS, Lee J, Shepherd L, Singer G, Ressler R, et al. Prospective, randomized, double-blind study comparing singleagent antibiotic therapy, ciprofloxacin, to combination antibiotic therapy in open fracture wounds. J Orthop Trauma. 2000;14(8):529-33. [PubMed: 11149497].

18. Joseph TN, Chen AL, Di Cesare PE. Use of antibiotic-impregnated ce- ment in total joint arthroplasty. J Am Acad Orthop Surg. 2003;11(1):3847. [PubMed: 12699370].

19. Klemm KW.Antibiotic bead chains. Clin Orthop Relat Res. 1993(295):6376. [PubMed: 8403672].

20. Ostermann PA, Seligson D, Henry SL. Local antibiotic therapy for severe open fractures. A review of 1085 consecutive cases. J Bone Joint Surg Br. 1995;77(1):93-7. [PubMed: 7822405].

21. Noel SP, Courtney H, Bumgardner JD, Haggard WO. Chitosan films: a potential local drug delivery system for antibiotics. Clin Orthop Relat Res. 2008;466(6):1377-82. doi: 10.1007/s11999-008-0228-1. [PubMed: 18421540].

22. Orhan Z, Cevher E, Mulazimoglu L, Gurcan D, Alper M, Araman A, et al. The preparation of ciprofloxacin hydrochloride-loaded chitosan and pectin microspheres: their evaluation in an animal osteomyelitis model. J Bone Joint Surg Br. 2006;88(2):270-5. doi: 10.1302/0301620X.88B2.16328. [PubMed: 16434537].

23. Smith JK, Bumgardner JD, Courtney HS, Smeltzer MS, Haggard WO Antibiotic-loaded chitosan film for infection prevention: A preliminary in vitro characterization. J Biomed Mater Res B Appl Biomater. 2010;94(1):203-11. doi: 10.1002/jbm.b.31642. [PubMed: 20524196].

24. El-Husseiny M, Patel S, MacFarlane RJ, Haddad FS. Biodegradable antibiotic delivery systems. J Bone Joint Surg Br. 2011;93(2):151-7. doi: 10.1302/0301-620X.93B2.24933. [PubMed: 21282751].

25. Noel SP, Courtney HS, Bumgardner JD, Haggard WO. Chitosan sponges to locally deliver amikacin and vancomycin: a pilot in vitro evaluation. Clin Orthop Relat Res. 2010;468(8):2074-80. doi: 10.1007/s11999-010-1324-6. [PubMed: 20352389].

26. Muzzarelli R, Tarsi R, Filippini O, Giovanetti E, Biagini G, Varaldo PE. Antimicrobial properties of N-carboxybutyl chitosan. Antimicrob Agents Chemother. 1990;34(10):2019-23. [PubMed: 2291669].

27. Aimin C, Chunlin H, Juliang B, Tinyin Z, Zhichao D. Antibiotic loaded chitosan bar. An in vitro, in vivo study of a possible treatment for osteomyelitis. Clin Orthop Relat Res. 1999(366):239-47. [PubMed: 10627741]. 